OPEN ACCESS

Edited by:

Zhang Sheng Jian,

Fudan University, China

Reviewed by:

Alessandro De Vita,

Istituto Scientifico Romagnolo per 10

Studio e il Trattamento dei Tumor

(IRCCS), Italy

Massimo Galia,

University of Palermo, Italy

${ }^{*}$ Correspondence:

Bin Song

songlab_radiology@163.com

Specialty section: This article was submitted to

Cancer Imaging and

Image-directed Interventions,

a section of the journal

Frontiers in Oncology

Received: 25 September 2021 Accepted: 30 November 2021 Published: 17 December 2021

Citation:

Che F, Yang CW, Hu X, Li Q

Wei Y, Liu Xj and Song B (2021)

Massive Calcified Epithelioid

Hemangioendothelioma With

Multifocal Involvement: An

Imaging Diagnosis Dilemma

and a Rare Case Report.

Front. Oncol. 11:782970.

doi: 10.3389/fonc.2021.782970

\section{Massive Calcified Epithelioid Hemangioendothelioma With Multifocal Involvement: An Imaging Diagnosis Dilemma and a Rare Case Report}

\author{
Feng Che ${ }^{1}$, Cai wei Yang ${ }^{1}$, Xue Hu ${ }^{1}$, Qian $\mathrm{Li}^{1}$, Yi Wei ${ }^{1}, \mathrm{Xi}$ jiao $\mathrm{Liu}^{2}$ and Bin Song ${ }^{2 *}$ \\ ${ }^{1}$ West China School of Medicine, West China Hospital, Sichuan University, Chengdu, China, ${ }^{2}$ Department of Radiology, \\ West China Hospital, Sichuan University, Chengdu, China
}

Epithelioid hemangioendothelioma (EHE) is a rare malignant vascular tumor that develops from vascular endothelial or pre-endothelial cells. More than $60 \%$ patients have singleorgan involvement, and involvement of multiple organs including the liver, lungs, and bones is extremely rare. The typical radiographic features of EHE include multiple small nodules in both lungs, which are usually located near small- and medium-sized blood vessels and the bronchi, and solitary, multiple, or diffuse lesions located at the hepatic periphery, spreading within the branches of the portal and hepatic veins. Radiologic calcification has been rarely reported in the literature. Here, we firstly described a case of a 53-year-old woman with EHE who presented with lungs, liver, bone, and right hilar lymph node involvement, manifesting as massive calcification on computed tomography. This case reminds physicians that EHE may present with unusual imaging manifestations, like massive calcification, and should be considered during the diagnostic process.

Keywords: epithelioid hemangioendothelioma, multifocal involvement, calcification, computed tomography, imaging diagnosis

\section{INTRODUCTION}

Epithelioid hemangioendothelioma (EHE) is a rare vascular neoplasm with a low-to-intermediate risk of malignancy which was first described in 1975 by Dail and Liebow (1). EHE originates from vascular endothelial cells or pre-endothelial cells, with a prevalence of less than one in one million that the current reported literature is limited to case reports. More than $60 \%$ patients have singleorgan involvement, most commonly, the liver (34\%), followed by the bones (21\%) and lungs (19\%) (2). And the involvement of multiple organs is extremely rare. Pulmonary epithelioid hemangioendothelioma(PEH) typically appear as perivascular nodules $1-2 \mathrm{~cm}$ in size throughout the bilateral lung fields, with a lower lobe predominance (3). Hepatic epithelioid hemangioendothelioma(HEH) commonly demonstrates multifocal nodular presentation, with computed tomography (CT) demonstrating clear margins (4). However, radiologic calcification has been rarely reported in the literature. Here, we have reported a very rare case of multifocal EHE presenting as massive calcifications on CT. The imaging features of this case-multiple nodules and 
masses accompanied by diffuse and massive calcifications in both lungs and liver as well as involvement of the bone and right hilar lymph nodes-are extremely rare. To the best of our knowledge, this is the first report of an EHE presenting as massive calcification radiologically, with multifocal involvement of the lungs, liver, and bones, which is also rare.

\section{CASE PRESENTATION}

A 53-year-old woman was referred to our clinic with waist and back pain and numbness of the lower limbs for more than 1 month. The pain was not related to her posture and became more prominent when she moved. She had a medical history of lumbar disc herniation and no history of trauma. On initial evaluation, her vital signs were stable. Apart from the pain of the waist and back, physical examination revealed unremarkable findings. Routine blood tests were obtained. Further, liver function tests revealed normal results. The blood CA199, CA125, CEA, and AFP levels were also within normal limits.

Computed tomography of the chest revealed scattered pulmonary nodules with calcifications associated with a soft tissue mass measuring $3.3 \mathrm{~cm} \times 2.4 \mathrm{~cm}$ and without pleural thickening at the superior lobe of the right lung (Figure 1) (SOMATOM definition, Siemens Healthcare, Erlangen, Germany; tube voltage, 100-120 kVp; tube current, $450 \mathrm{~mA}$; slice thickness, $0.625 \mathrm{~mm}$; pitch, 0.992:1; rotation speed: $0.5 \mathrm{~s} /$ rot; ASIR-V:30\%.). Enlarged lymph nodes of the right hilar were also evident. Abdominal contrast-enhanced CT revealed diffuse lesions with massive calcifications in the liver, which shows faint peripheral enhancement in the arterial phase and low enhancement in the portal phase (Iopromide Injection, Bayer Pharma AG; the arterial phase and portal venous phase were obtained at $25 \mathrm{~s}$ and $60 \mathrm{~s}$ after contrast injection.). The largest lesion measuring $10.2 \mathrm{~cm} \times 5.9 \mathrm{~cm}$ was located in the right lobe of the liver and (Figure 2). CT examination also revealed osteolytic lesions with a massive thick sclerotic rim in the right second rib, 11th thoracic vertebra, and first lumbar spine. Bone scintigraphy with $99 \mathrm{mTc}$-methylene diphosphonate showed multiple hypermetabolic activities in the involved bones (Figure 3). Cerebral magnetic resonance imaging (MRI) revealed no anomalies. The patient underwent transthoracic needle biopsy of the largest pulmonary lesion located in the right superior lobe. Histopathological analysis revealed epithelioid cells arranged in a glandular pattern with clear cytoplasm (Figure 4). Immunohistochemical staining showed that the neoplastic cells were positive for CD31, CD34, CAMTA1, and EMA, but negative for ERG, TFE3, PCK, and desmin, with a Ki-67 index rate of $10 \%$. Histopathological examination indicated a rare low-grade malignant vascular neoplasm, confirming the diagnosis of EHE.

Considering the multiple intra-pulmonary, right hilar lymph node, liver, and bone metastases, the patient was treated with chemotherapy with paclitaxel liposome $\left(240 \mathrm{mg} / \mathrm{m}^{2}\right.$; day 1$)$ and carboplatin $\left(550 \mathrm{mg} / \mathrm{m}^{2}\right.$; day 1$)$. At 8 months, the patient had completed four cycles of combination therapy. There were no changes in the patient's disease status on CT at the 8-month follow-up visit.

\section{DISCUSSION}

EHE is a low-grade malignant vascular tumor that is usually located in soft tissues or deep internal structures. The age at diagnosis ranges from 3 years to 86 years, with a peak incidence in middle-aged adults and a mild female predilection (male: female: 1:4) $(5,6)$. Most patients have nonspecific symptoms on initial presentation with generally indolent clinical activity, but can sometimes demonstrate aggressive clinical behavior (7). Diagnosis of EHE depends on a combination of unique

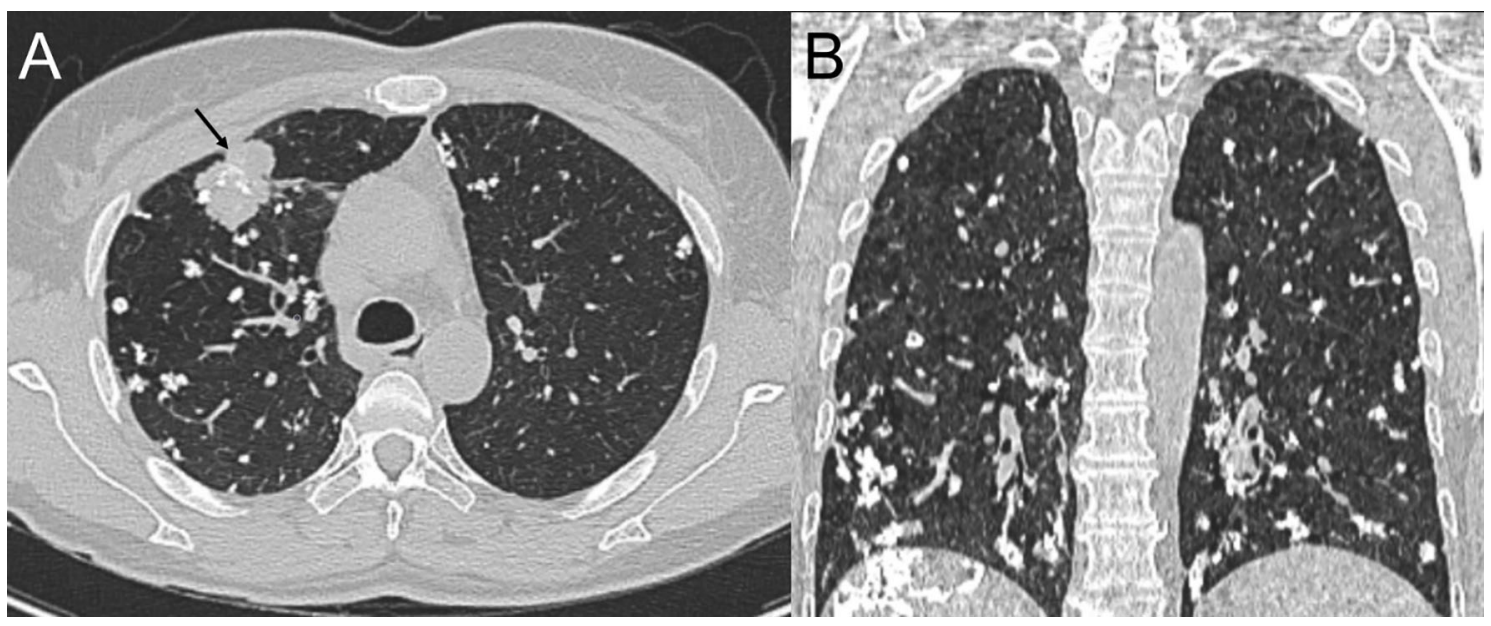

FIGURE 1 | Computed tomography (CT) scan of the chest, lung window, axial view (A) and coronal view (B), showing a mass in the right superior lobe (black arrow) and bilateral soft tissue nodules in the lungs with diffuse calcifications adjacent to the bronchioles and small- and medium-sized vessels. 


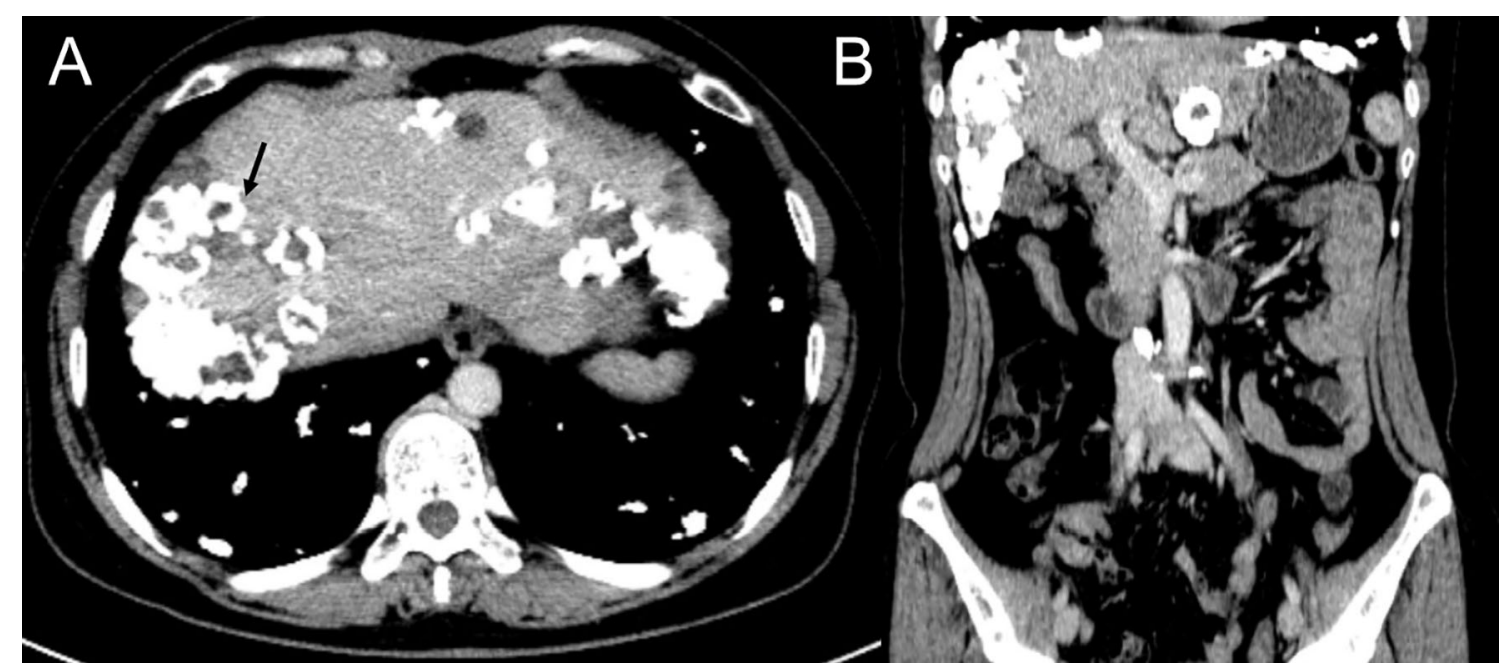

FIGURE 2 | Contrast enhanced CT scan of the abdomen, portal phase, axial view (A) and coronal view (B), showing scattered calcified nodules and mass in the liver with the biggest lesion located in the right lobe (black arrow).

histologic, immunohistochemical, and molecular characteristics since there is nonspecific imaging and the differential diagnosis for EHE is broad. Usually, it is histologically characterized by epithelioid tumor cells that are spread throughout the myxohyaline stroma. The tumor cells are immunohistochemically positive for vascular endothelial markers, including CD31, CD34, cytokeratin, vimentin, and factor VIII antigen. And the Ki-67 index rate of $>10 \%-15 \%$ has been shown to be a more aggressive feature $(3,8)$. Nodules measuring $3 \mathrm{~cm}$ were also considered to demonstrate a worse survival (9). Our patient had a pulmonary mass measuring $3.3 \mathrm{~cm}$ and a liver mass measuring $10.2 \mathrm{~cm}$ as well as bone involvement, further immunohistochemical staining showed Ki-67 index rate of $10 \%$. What is more remarkable is its unique radiographic findings, which appears to be a challenge for clinician to give a consideration of this rare disease.

The typical radiographic findings of $\mathrm{PEH}$ are multiple small nodules in both lungs, which are usually located near small- or medium-sized blood vessels and the bronchi. Most PEH lesions are less than $1 \mathrm{~cm}$ in diameter, but occasionally, lesions up to 2 $\mathrm{cm}$ can occur. This is the first case report of a $\mathrm{PEH}$ with rare radiologic features including numerous distinct calcified nodules with largest mass measuring $3.3 \mathrm{~cm}$. This presentation can be easily misdiagnosed as other lung diseases such as disseminated tuberculosis, metastasis (such as metastases secondary to osteosarcoma, mucin-producing carcinoma, thyroid malignancy, and treated metastatic choriocarcinoma), and pulmonary amyloidosis. Radiologic calcifications in $\mathrm{PEH}$ are rare, but histological examinations often show nodules with calcified and ossified necrotic centers on microscopy (10). In most cases, patients develop calcifications 10-20 years after diagnosis, the majority of which tend to be punctate calcifications. Our patient was diagnosed with lumbar disc herniation 2 years prior and showed no abnormalities in the lungs at that time. The mechanism of calcification in these nodules is multifactorial which is not precisely understood.
Further research is needed to clarify whether this is due to the disease itself or individual heterogeneity.

In EHE, liver is more frequently involved than lung. $\mathrm{HEH}$ can be classified as solitary, multiple, or diffuse. The multifocal nodules generally spread within the branches of the portal and hepatic veins and, most commonly, at the periphery. Intratumor calcification, capsular retraction, halo sign, and lollipop sign have been reported as relevant imaging features for distinguishing $\mathrm{HEH}$ on CT or MRI. Intratumor calcification is an inconsistent but suggestive feature of $\mathrm{HEH}$ and is only seen in $12.7 \% \mathrm{HEH}$ cases $(11,12)$. The presence of coalescent lesions is considered a risk factor that is often associated with hepatic and/or portal vein inversion (13-15). Our patient presented with extremely rare imaging features of scattered nodules/masses with extensive calcifications accompanied by coalescence lesions. The tumors mainly located in the periphery without actual bulging of the liver capsule, which is assumed to be a radiologic characteristic of $\mathrm{HEH}$ (16). Differential diagnosis of $\mathrm{HEH}$ is difficult and should be distinguished from multifocal metastases (such as metastases of breast, adrenal gland, or colon tumors), multiple sclerosing hemangioma, hepatocellular carcinoma (HCC) with hepatic metastases, intrahepatic parasitic or bacterial infections, and others. Typical imaging findings are important for pre-treatment diagnosis, but a combination of clinical examination, medical history, laboratory and pathological assessments are essential for diagnosis. Occasionally, immunohistochemical and molecular biology analysis is needed for that the tumor can be mistaken for HCC or metastatic carcinoma, cholangiocarcinoma, angiosarcoma or undifferentiated sarcomas on histological examination.

Bone EHE accounts for less than $1 \%$ of all primary bone neoplasms. Cases occurring in the spine region are especially rare as long tubular bones are more commonly affected (17). The typical radiographic characteristic of bone EHE is an osteolytic lesion with a thin sclerotic rim. Periosteal reactions and matrix calcification are uncommon $(3,18)$. Our case showed massive high-attenuation lesions in the involved bones with an apparent thick rim that could 


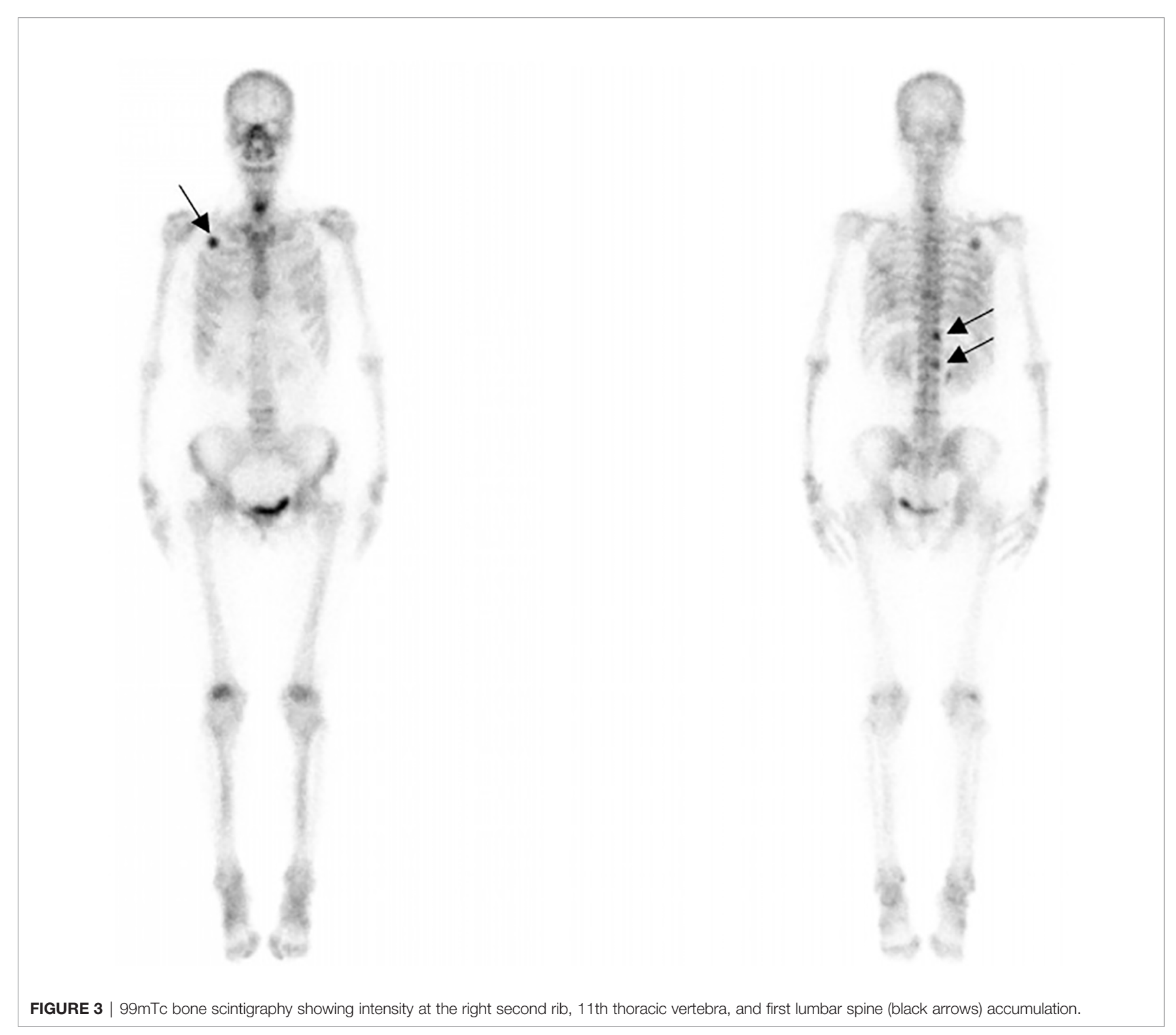

not be easily differentiated as calcification or ossification. However, high-attenuation lesions are similarly identified with calcification in lung and liver lesions. Multifocal EHE of the bones can be easily misdiagnosed as metastatic tumors, multiple myelomas, or brown tumors owing to the overlapping imaging features, while previous research has indicated that the clustering of multiple lesions in the same anatomic region can help with the diagnosis of a vascular tumor. For bone EHE, whole-body scintigraphy has the advantage of showing the distributional characteristics of multiple lesions. Earlier detection of silent lesions is possible owing to its high sensitivity $(19,20)$, although the final diagnosis depends on histopathology and immunochemical staining results.

The diagnosis of EHE can be challenging, and treatment options are not standardized. There is limited clinical data to guide treatment choices due to its rarity. Consequently, very few therapeutic options are available and treatment throughout the literature review has been based on individual basis. Surgical resection can achieve good outcomes for patients with a limited number of lesions and no metastases. Chemotherapy or immunotherapy can be considered in patents with asymptomatic metastatic disease $(21,22)$. Some studies have proven that radiotherapy can help patients with exclusive bone presentations achieve local pain control and better quality of life when combined with bone surgery $(23,24)$. There are several cases of cancer regression or remission achieved in patients treated with conventional chemotherapy drugs such as carboplatin, etoposide, and paclitaxel (25). Our patient opted for chemotherapy with paclitaxel liposomes and carboplatin, both of which are cell cyclenonspecific drugs, due to its disseminated and unresectable characteristic. Eight months after treatment, the discomfort caused by lower back distress disappeared. However, there was no change in the patient's disease status on CT. Treatment evidence for this rare disease is limited to small trials and case series, more clinical trials are needed to identifying new treatment targets. 


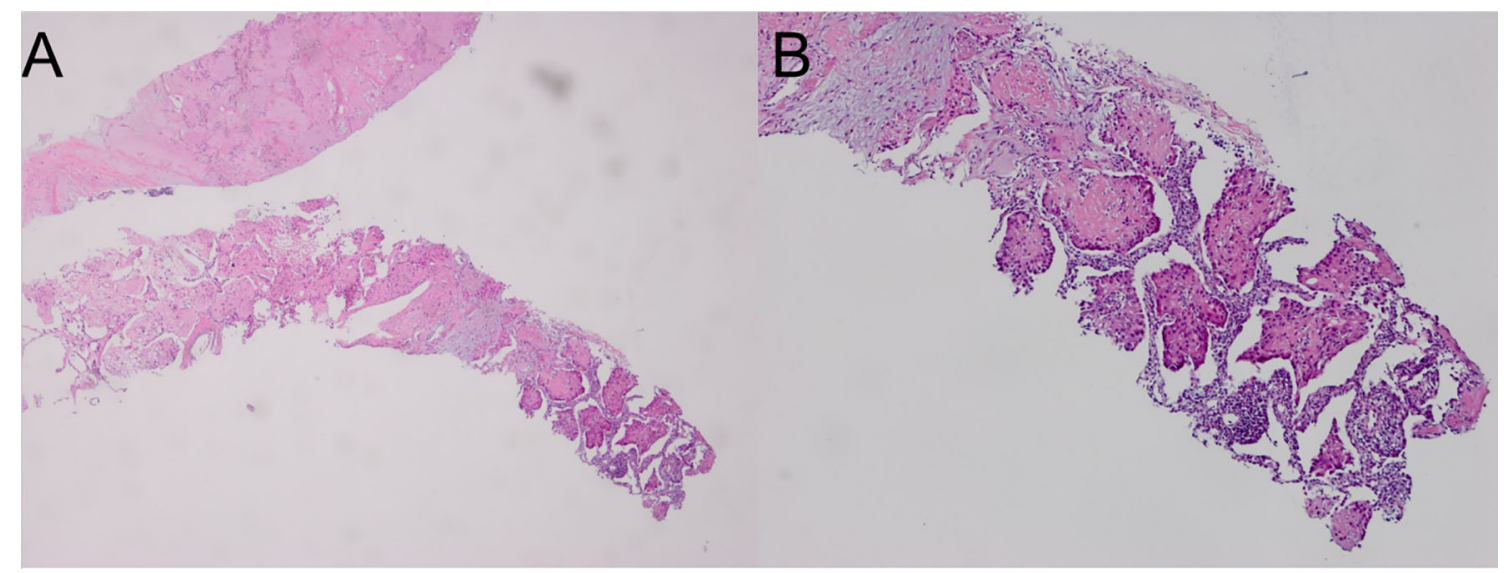

FIGURE 4 | Hematoxylin and eosin staining of the lung biopsy sample showing scattered spindle-shaped tumor cells and epithelioid tumor cells in a myxohyaline stroma. (A; hematoxylin and eosin, $\times 40$ ); higher magnification of the expanded sinusoids lined by large epithelioid tumor cells (B; hematoxylin and eosin, $\times 100)$.

\section{CONCLUSION}

In conclusion, this case illustrates a rare manifestation of EHE with multiple organ involvement. Each lesion manifested with an uncommon radiological finding with extensive calcification. Since there are no specific radiological signs, we should be aware of the possible radiological presentations of EHE, which may help with preoperative diagnosis and clinical treatment planning.

\section{DATA AVAILABILITY STATEMENT}

The original contributions presented in the study are included in the article/supplementary material. Further inquiries can be directed to the corresponding author.

\section{REFERENCES}

1. Dail DH, Liebow AA, Gmelich JT, Friedman PJ, Miyai K, Myer W, et al. Intravascular, Bronchiolar, and Alveolar Tumor of the Lung (IVBAT). An Analysis of Twenty Cases of a Peculiar Sclerosing Endothelial Tumor. Cancer (1983) 51:452-64. doi: 10.1002/1097-0142(19830201)51:3<452::AIDCNCR2820510317>3.0.CO;2-M

2. Lau K, Massad M, Pollak C, Rubin C, Yeh J, Wang J, et al. Clinical Patterns and Outcome in Epithelioid Hemangioendothelioma With or Without Pulmonary Involvement: Insights From an Internet Registry in the Study of a Rare Cancer. Chest (2011) 140:1312-8. doi: 10.1378/chest.11-0039

3. Sardaro A, Bardoscia L, Petruzzelli MF, Portaluri M. Epithelioid Hemangioendothelioma: An Overview and Update on a Rare Vascular Tumor. Oncol Rev (2014) 8:259. doi: 10.4081/oncol.2014.259

4. Alomari AI. The Lollipop Sign: A New Cross-Sectional Sign of Hepatic Epithelioid Hemangioendothelioma. Eur J Radiol (2006) 59:460-4. doi: 10.1016/j.ejrad.2006.03.022

5. Woo JH, Kim TJ, Lee KS, Kim TS, Kim BT. Epithelioid Hemangioendothelioma in the Thorax: Clinicopathologic, CT, PET, and Prognostic Features. Med (Baltimore) (2016) 95:e4348. doi: 10.1097/MD.0000000000004348

6. Liu K, Xie P, Peng W, Zhou Z. The Computed Tomographic Findings of Pulmonary Epithelioid Hemangioendothelioma. Radiol Med (2014) 119:70513. doi: 10.1007/s11547-013-0376-6

\section{ETHICS STATEMENT}

The studies involving human participants were reviewed and approved by the West China Hospital of Sichuan University. The patients/participants provided their written informed consent to participate in this study.

\section{AUTHOR CONTRIBUTIONS}

FC and $\mathrm{XH}$ acquired the data. YW, $\mathrm{CY}$, and QL analyzed and interpreted the data. FC and $\mathrm{CY}$ conducted the radiological analysis of MRI and CT images, and FC prepared the manuscript. XL and BS revised the manuscript. All authors contributed to the article and approved the submitted version.

7. Lytle M, Bali SD, Galili Y, Bednov B, Murillo Alvarez RM, Carlan SJ, et al. Epithelioid Hemangioendothelioma: A Rare Case of an Aggressive Vascular Malignancy. Am J Case Rep (2019) 20:864-7. doi: 10.12659/ AJCR.915874

8. Dong K, Wang XX, Feng JL, Liu H, Zu KJ, Chang J, et al. Pathological Characteristics of Liver Biopsies in Eight Patients With Hepatic Epithelioid Hemangioendothelioma. Int J Clin Exp Pathol (2015) 8:11015-23.

9. Shibayama T, Makise N, Motoi T, Mori T, Hiraoka N, Yonemori K, et al. Clinicopathologic Characterization of Epithelioid Hemangioendothelioma in a Series of 62 Cases: A Proposal of Risk Stratification and Identification of a Synaptophysin-Positive Aggressive Subset. Am J Surg Pathol (2021) 45:61626. doi: 10.1097/PAS.0000000000001660

10. Cronin P, Arenberg D. Pulmonary Epithelioid Hemangioendothelioma: An Unusual Case and a Review of the Literature. Chest (2004) 125:789-93. doi: 10.1378/chest.125.2.789

11. Mehrabi A, Kashfi A, Fonouni H, Schemmer P, Schmied BM, Hallscheidt P, et al. Primary Malignant Hepatic Epithelioid Hemangioendothelioma: A Comprehensive Review of the Literature With Emphasis on the Surgical Therapy. Cancer (2006) 107:2108-21. doi: 10.1002/cncr.22225

12. Zhao XY, Rakhda MI, Habib S, Bihi A, Muhammad A, Wang TL, et al. Hepatic Epithelioid Hemangioendothelioma: A Comparison of Western and Chinese Methods With Respect to Diagnosis, Treatment and Outcome. Oncol Lett (2014) 7:977-83. doi: 10.3892/ol.2014.1847 
13. Fukayama M, Nihei Z, Takizawa T, Kawaguchi K, Harada H, Koike M. Malignant Epithelioid Hemangioendothelioma of the Liver, Spreading Through the Hepatic Veins. Virchows Arch A Pathol Anat Histopathol (1984) 404:275-87. doi: 10.1007/BF00694893

14. Clements D, Hubscher S, West R, Elias E, McMaster P. Epithelioid Haemangioendothelioma. A Case Report. J Hepatol (1986) 2:441-9. doi: 10.1016/S0168-8278(86)80055-1

15. Cobden I, Johri S, Terry G, Robinson KB, Bennett MK, Lendrum R. Hepatic Epithelioid Haemangioendothelioma: Difficult Name, Difficult Diagnosis? Postgrad Med J (1988) 64:128-31. doi: 10.1136/pgmj.64.748.128

16. Miller WJ, Dodd GD3rd, Federle MP, Baron RL. Epithelioid Hemangioendothelioma of the Liver: Imaging Findings With Pathologic Correlation. AJR Am J Roentgenol (1992) 159:53-7. doi: 10.2214/ajr.159.1.1302463

17. van IDGP, Bovée J. Vascular Tumors of Bone: The Evolvement of a Classification Based on Molecular Developments. Surg Pathol Clin (2017) 10:621-35. doi: 10.1016/j.path.2017.04.003

18. Wenger DE, Wold LE. Malignant Vascular Lesions of Bone: Radiologic and Pathologic Features. Skeletal Radiol (2000) 29:619-31. doi: 10.1007/ s002560000261

19. Zhang H, Fu Y, Ye Z. Bone Multicentric Epithelioid Hemangioendothelioma of the Lower and Upper Extremities With Pulmonary Metastases: A Case Report. Oncol Lett (2015) 9:2177-80. doi: 10.3892/ol.2015.3018

20. Dechambre SD, Coche EE, Roisin P, Van Eeckhout P, D'Odemont JP. Epithelioid Hemangioendothelioma With Solitary Bone Location Associated to Multiple Lung and Liver Lesions. A Case Report. Acta Radiol (1999) 40:217-9. doi: 10.3109/02841859909177742

21. Stacchiotti S, Miah AB, Frezza AM, Messiou C, Morosi C, Caraceni A, et al. Epithelioid Hemangioendothelioma, an Ultra-Rare Cancer: A Consensus Paper From the Community of Experts. ESMO Open (2021) 6:100170. doi: 10.1016/j.esmoop.2021.100170
22. Recine F, Bongiovanni A, Riva N, Fausti V, De Vita A, Mercatali L, et al. Update on the Role of Trabectedin in the Treatment of Intractable Soft Tissue Sarcomas. Onco Targets Ther (2017) 10:1155-64. doi: 10.2147/OTT.S127955

23. van Kasteren ME, van der Wurff AA, Palmen FM, Dolman A, Miseré JF. Epithelioid Haemangioendothelioma of the Lung: Clinical and Pathological Pitfalls. Eur Respir J (1995) 8:1616-9. doi: 10.1183/09031936.95.08091616

24. Aquilina K, Lim C, Kamel MH, Marks CJ, O'Sullivan MG, Keohane C. Epithelioid Hemangioendothelioma of the Spine. Report of Two Cases. J Neurosurg Spine (2005) 3:393-9. doi: 10.3171/spi.2005.3.5.0393

25. Ye B, Li W, Feng J, Shi JX, Chen Y, Han BH. Treatment of Pulmonary Epithelioid Hemangioendothelioma With Combination Chemotherapy: Report of Three Cases and Review of the Literature. Oncol Lett (2013) 5:1491-6. doi: 10.3892/ol.2013.1217

Conflict of Interest: The authors declare that the research was conducted in the absence of any commercial or financial relationships that could be construed as a potential conflict of interest.

Publisher's Note: All claims expressed in this article are solely those of the authors and do not necessarily represent those of their affiliated organizations, or those of the publisher, the editors and the reviewers. Any product that may be evaluated in this article, or claim that may be made by its manufacturer, is not guaranteed or endorsed by the publisher.

Copyright (C) 2021 Che, Yang, Hu, Li, Wei, Liu and Song. This is an open-access article distributed under the terms of the Creative Commons Attribution License (CC BY). The use, distribution or reproduction in other forums is permitted, provided the original author(s) and the copyright owner(s) are credited and that the origina publication in this journal is cited, in accordance with accepted academic practice. No use, distribution or reproduction is permitted which does not comply with these terms. 